\title{
Clinical Trial Protocol: Randomized Controlled Trial of Cancer Pain Monitoring System (CAPAMOS) in Patients with Advanced Cancer
}

\author{
Shiori Yoshida ${ }^{1}$, Fumiko Sato ${ }^{1}$, Keita Tagami², Shin Takahashi ${ }^{3}$ \\ ${ }^{1}$ Department of Oncology Nursing, Tohoku University Graduate School of Medicine, Sendai, Japan \\ ${ }^{2}$ Department of Palliative Medicine, Tohoku University Graduate School of Medicine, Sendai, Japan \\ ${ }^{3}$ Department of Medical Oncology, Tohoku University Hospital, Sendai, Japan \\ Email: ^shiori.y@med.tohoku.ac.jp
}

How to cite this paper: Yoshida, S., Sato, F., Tagami, K. and Takahashi, S. (2022) Clinical Trial Protocol: Randomized Controlled Trial of Cancer Pain Monitoring System (CAPAMOS) in Patients with Advanced Cancer. Open Journal of Nursing, 12, 113-124.

https://doi.org/10.4236/ojn.2022.122008

Received: January 6, 2021

Accepted: February 18, 2022

Published: February 21, 2022

Copyright $\odot 2022$ by author(s) and Scientific Research Publishing Inc. This work is licensed under the Creative Commons Attribution International License (CC BY 4.0).

http://creativecommons.org/licenses/by/4.0/

\begin{abstract}
Purpose: This randomized controlled trial (RCT) protocol was designed to evaluate the effectiveness of the Cancer Pain Monitoring System (CAPAMOS), a telenursing system designed to alleviate pain in ambulatory patients with advanced cancer. Methods: The study design involved a randomized controlled trial consisting of an intervention group using CAPAMOS and a control group that received usual care. At the time of enrollment, both groups will be given a questionnaire regarding issues, such as concerns related to cancer pain and self-management of opioid medication. Patients will then receive pain relief education based on the results of the questionnaire. Subjects in the control group will receive a pain diary and pain-relief pamphlet that acts as an educational tool. They could also call the outpatient clinic if they wanted to ask for a nursing consultation. The intervention group will be able to use CAPAMOS to manage their pain and receive video consultation with a telenurse, who is an oncology nursing specialist. Educational tools will be built into CAPAMOS. The study will be conducted for one month. The primary endpoint of this study was to calculate the Japanese Brief Pain Inventory score. The secondary endpoints were to assess the opioid medication self-management, Barriers Questionnaire, Japanese EuroQol 5-dimensions 5-level, and economic evaluation. Assessment items will be evaluated at registration and 2 and 4 weeks after registration. Conclusions: A RCT based on this protocol is expected to validate the efficacy of telenursing using CAPAMOS in patients with advanced cancer and pain.
\end{abstract}

\section{Keywords}

Telenursing, Cancer Pain, Advanced Cancer Patients, Symptom Management, Patient Care, Randomized Controlled Trial 


\section{Introduction}

Approximately $60 \%$ of outpatients with advanced cancer experience pain, and of those, approximately $20 \%$ undergo moderate-to-severe pain [1]. Cancer-related pain becomes more complex as cancer progresses, resulting in deterioration in the quality of life (QOL). In Japan, with the advent of a super-aging society and increasing social security costs, early discharge from hospitals is being promoted [2], and the place of treatment for patients with advanced cancer is shifting from hospitals to homes. Patients may have difficulty coping with distressing symptoms that appear at home.

We have previously investigated the relationship between self-management of cancer-related pain and pain in outpatients with advanced cancer and found that those who were unable to take their medication on time had a greater disruption in their life as a result of pain [3]. Medical professionals need to know more about the life situations in which patients are unable to take their medication on time. In addition, the presence of medical personnel, who can be consulted without anxiety, is an important factor in pain relief for patients with advanced cancer at home [4]. In a society where early discharge from hospitals is being increased, support of patients with advanced cancer at home is essential by providing follow-up by medical professionals.

In recent years, the Japanese government has been promoting telemedicine to support medical care [5] [6] [7]. Information and communication technology is expected to be used for symptoms' management of patients with advanced cancer, who spend their time at home. The benefits of telenursing to support outpatient cancer patients include reduced pain and distress symptoms [8] [9] [10] [11], improved QOL [8], reduced psychiatric symptoms [10], fewer emergency visits [11], and improved survival [11] [12]. The support provided through telenursing includes patients receiving effective and up-to-date information about symptoms management, as well as facilities to report and monitor their symptoms on a daily basis with their healthcare providers.

We developed the Cancer Pain Monitoring System (CAPAMOS) as a telenursing system to alleviate cancer pain. Patients can utilize the CAPAMOS to record pain and the administration of opioid medication daily to better understand their pain fluctuations. Patients will then be able to obtain accurate information on pain relief from the system. Furthermore, patients are expected to receive early pain relief care as a result of monitoring by an oncology nursing specialist. In our previous study [13], we evaluated the use of the CAPAMOS. The purpose of this study was to investigate the efficacy of the CAPAMOS in reducing pain in outpatients with advanced cancer.

\section{Methods}

\subsection{Conceptual Framework}

In this study, telenursing self-management support was based on the Tel-eNurse Practice Model (TNPM) and the model of symptom management (MSM), re- 
spectively.

The framework of telenursing practice in this study was the TNPM, in which the telenurse provides comprehensive care and self-management information to patients based on personal, health, and nursing relationships. In addition, telenurse collaborates with other professionals for health promotion and provides resources for health behavior changes [14] [15].

The framework for cancer pain relief was MSM, which consists of three interrelated concepts: "symptom experience", "management strategies" and "outcomes of symptom management" [16]. A patient's experience influences the strategies they adopt, and the outcomes of which affect their next experience.

The educational tool in CAPAMOS provides knowledge about pain and opioids as well as what to do when pain is not relieved. Therefore, patients could self-manage their pain. If pain is not relieved, the patient can consult a telenurse. If the pain level increases, the telenurse can check the patient's physical condition and provide educational guidance on how to manage pain.

\subsection{Study Design}

This was a randomized controlled trial (Figure 1) and the primary endpoint was the degree of cancer-related pain at the end of a month. The sample size for this study was calculated based on previous studies [17], so that the two-sided level

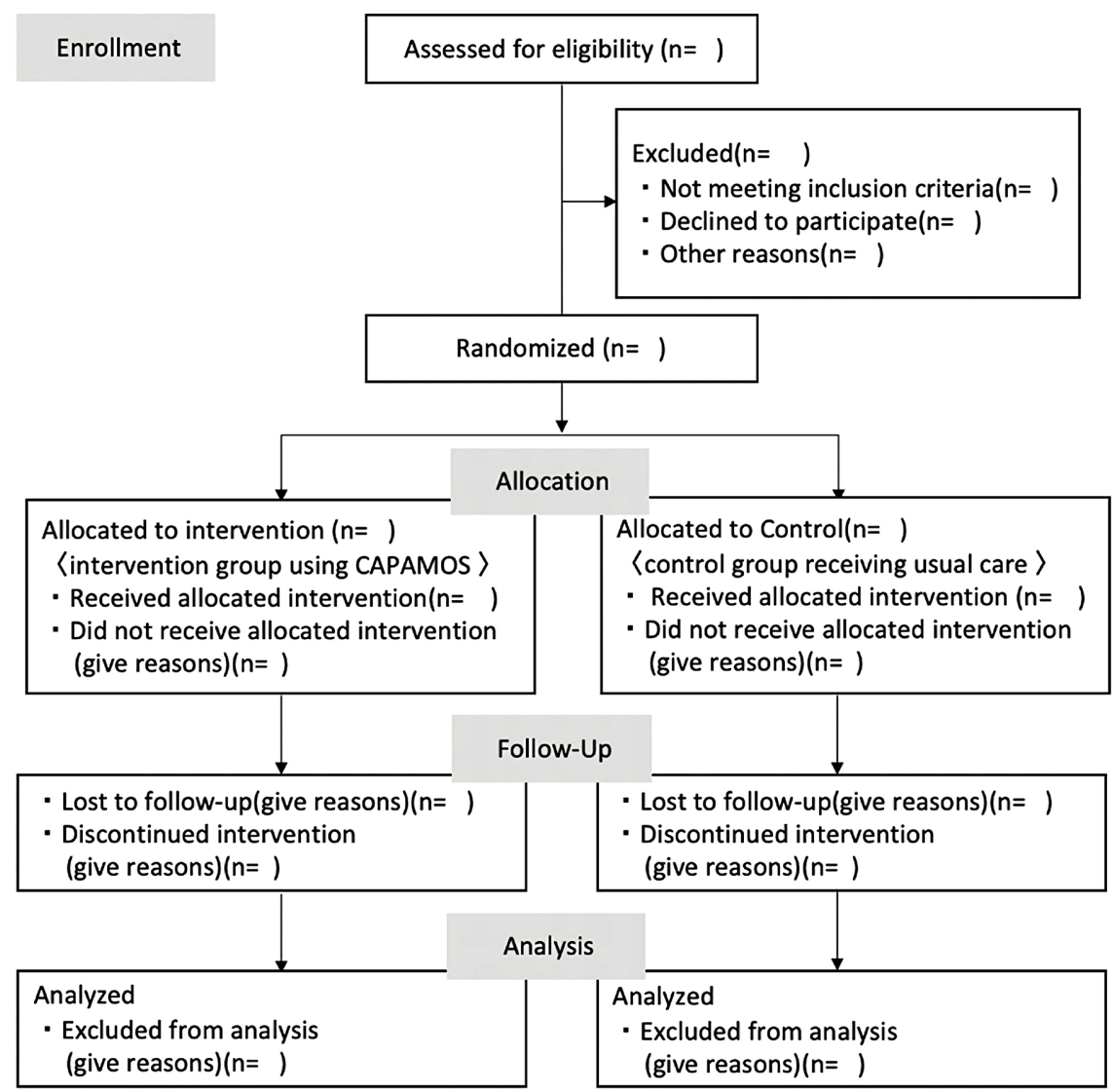

Figure 1. Participant recruitment and sample sizes. 
of significance was fixed at 0.5 , the power was set at 0.8 , and a dropout rate of $20 \%$ was considered. A sample size of 48 was selected, with 24 participants in each group.

Patients will be assigned to an intervention group involving the use of CAPAMOS or a control group receiving usual care. At the time of enrollment, both groups will be given a questionnaire regarding issues, such as concerns related to cancer pain and opioid medication, and self-management of opioid medication. They will then receive pain relief education based on the results of the questionnaire. Subjects in the control group will receive a pain diary and pain-relief pamphlet that acts as an educational tool. They could also call the outpatient clinic if they wanted to ask for a nursing consultation. The intervention group will be able to use CAPAMOS to manage their pain and receive video consultation with a telenurse. Educational tools were built into CAPAMOS.

The study will be conducted between October 2019 and June 2025, with a one-month participation period for each subject. The research subjects were those who had received the research explanation from the researcher and signed the consent form. Data concerning the study variables will be collected at enrollment, at two weeks, and four weeks after enrollment. Randomization assigns numbers in a 1:1 ratio to the order in which they are recruited using a random number table. Double-blinding cannot be implemented because of the medium used in the intervention. Depending on the distribution of CAPAMOS, patients can know whether to intervene. The healthcare provider can determine if this is an intervention because the results of the monitoring may be reported by the telenurse.

\subsection{Content of Evaluation}

These are the evaluation variables for this study (Table 1).

The primary endpoint of this study was the Japanese Brief Pain Inventory (BPI-J) short form [18]. Patients were asked to rate their pain (worst, least, average, and now) in the last 24 hours. The four categories associated with subjective pain were graded on an 11-point scale from "No pain ( 0 points)" to "Pain as bad as you can imagine (10 points)" such that the higher the score, the more severe the pain. This study used the following aspects of pain: worst pain and average pain. Using the same type of 0 to 10 scale, patients were also asked to rate separately how their pain interfered with several life domains, including enjoyment of life, activity, walking, mood, sleep, work, and relationships with others. Interference with daily life caused by pain was graded on an 11-point scale from "Does not interfere (0 points)" to "Completely interferes (10 points)". The average score of the seven items was used to calculate the effect of pain on daily life; the higher the score, the more severe the effect of pain in their daily lives. Another BPI-J supplementary item was used to ask patients to rate the level of pain relief due to treatments or medications in the last 24 hours. This was graded on an 11-point scale from "No Relief (0\%)" to "Complete Relief (100\%)", with a higher percentage being an indication of better pain relief. 
Table 1. Variables to be used in the study.

\begin{tabular}{|c|c|c|}
\hline $\begin{array}{c}\text { Scales and } \\
\text { Medical Records }\end{array}$ & Subscales and variables & $\begin{array}{l}\text { At the time } \\
\text { of survey }\end{array}$ \\
\hline $\begin{array}{l}\text { the Japanese Brief Pain } \\
\text { Inventory Short Form }\end{array}$ & $\begin{array}{l}\text { Worst pain } \\
\text { Average pain } \\
\text { Interference with daily life } \\
\text { "In the last } 24 \text { hours, how much relief have pain } \\
\text { treatments or medications provided?" }\end{array}$ & $\begin{array}{l}\text { Enrollment } \\
2 \text { weeks } \\
4 \text { weeks }\end{array}$ \\
\hline $\begin{array}{l}\text { the Opioid } \\
\text { Self-Management } \\
\text { Scale for Advanced } \\
\text { Cancer Patients }\end{array}$ & $\begin{array}{l}\text { Managing opioids and coping with pain } \\
\text { Talking to healthcare providers } \\
\text { Talking to family and friends } \\
\text { Recording pain and opioid use } \\
\text { Living with the disease } \\
\text { Understanding pain characteristics }\end{array}$ & $\begin{array}{l}\text { Enrollment } \\
4 \text { weeks }\end{array}$ \\
\hline $\begin{array}{l}\text { the Japanese version } \\
\text { of the Barriers } \\
\text { Questionnaire }\end{array}$ & $\begin{array}{l}\text { Fatalism } \\
\text { Addiction } \\
\text { side effects } \\
\text { Progression } \\
\text { Tolerances } \\
\text { distraction }\end{array}$ & $\begin{array}{l}\text { Enrollment } \\
4 \text { weeks }\end{array}$ \\
\hline $\begin{array}{l}\text { the Japanese version } \\
\text { of the EQ-5D-5L }\end{array}$ & $\begin{array}{l}\text { Degree of mobility } \\
\text { Self-care } \\
\text { Usual activities } \\
\text { Pain/Discomfort } \\
\text { Anxiety/Distraction }\end{array}$ & $\begin{array}{l}\text { Enrollment } \\
4 \text { weeks }\end{array}$ \\
\hline \multirow[b]{2}{*}{ Medical records Items } & $\begin{array}{l}\text { Age, sex, type of cancer, stage, past history, and } \\
\text { treatment }\end{array}$ & Enrollment \\
\hline & $\begin{array}{l}\text { Data on prescribed medications } \\
\text { Status of consultation with specialists on pain relief } \\
\text { Costs due to worsening }\end{array}$ & $\begin{array}{l}\text { Enrollment } \\
2 \text { weeks } \\
4 \text { weeks }\end{array}$ \\
\hline
\end{tabular}

The secondary endpoint was the Opioid Self-Management Scale for Advanced Cancer Patients (OSSA). This scale was developed based on the self-management of opioid analgesics for cancer pain relief at home in patients with advanced cancer [4]. The scale consists of 33 items divided into six subscales: managing opioids and coping with pain, talking to healthcare providers, talking to family and friends, recording pain and opioid use, living with the disease, and understanding pain characteristics. A five-point scale from "No (0)" to "Yes (4)" was used to rate these items, with higher scores indicating better management skills.

The third assessment scale was the Japanese version of the Barriers Questionnaire (BQ) [19]. The BQ consists of 21 items divided into six subscales: fatalism, addiction, side effects, progression, tolerances and distraction. The items are graded on a six-point scale from "not at all (0 points)" to "very much (5 points)", with higher scores indicating stronger concerns.

The fourth assessment scale was the Japanese version of the EQ-5D-5L [20], which consists of 25 items divided into five subscales: degree of mobility, self-care, 
usual activities, pain/discomfort, and anxiety/distraction.

Data on age, sex, cancer type and stage, medical history, and treatment history were collected from medical records at the time of enrollment. In addition, data on prescribed medications, status of consultation with specialists on pain relief, and costs due to worsening symptoms (number of emergency visits and hospitalizations, hospitalization costs, opportunity losses related to labor, and prescription costs) will also be obtained for the study period.

\subsection{Participants}

Patients attending the outpatient departments of medical oncology, palliative medicine, breast surgery, and respiratory medicine at Tohoku University Hospital were enrolled in this study. Patients' eligibility criteria were as follows: 1) diagnosis of advanced cancer; 2) regular administration of opioids for cancer pain relief; 3) average pain $\geq 3$ in 24 hours, as measured by the numerical rating scale (NRS); 4) survival rate of at least 1 month; 5) $\geq 20$ years old; 6) notification of the disease name; 7) consent to participate. The exclusion criteria for the study were as follows: 1) regular use of opioids for purposes unrelated to cancer pain, such as dyspnea, and 2) physical, mental, or cognitive abilities that interfere with participation in the study.

The criteria for discontinuation of the research intervention were as follows: 1) excessive mental burden on the subject, 2) adverse events, and 3) withdrawal of consent from the subject.

\subsection{Intervention Program: CAPAMOS}

CAPAMOS was developed by three oncology researchers: one oncology nurse specialist, one oncology pharmacotherapy specialist, and one palliative medicine specialist. The draft of the educational tool was prepared by an oncology nurse specialist, an oncology pharmacotherapy specialist, and a palliative medicine specialist. Based on the draft, the content and surface validity of the educational tool were evaluated by two oncology nurse specialists, three nurses who attended the end-of-life nursing education consortium-Japan (ELNEC-J), three nursing researchers, and four laypeople in their 30s, 40s, 50s, and 60s. Previous trials have demonstrated the usability of CAPAMOS, with its clear structure and reliable content for patients [13].

Patients entered details of their daily medication and pain levels into CAPAMOS to manage their condition every day. When they feel unwell or need advice, they can consult a telenurse or learn about symptom management using educational tools. The structure of CAPAMOS (Figure 2) was based on the findings of previous studies [11] [17] [21]. The CAPAMOS consists of the following features: 1) "Cancer Pain and Pain Management Precautions", which is an educational tool for cancer pain palliation; 2) "Medication Check", in which medication status is entered; 3) "Physical Condition Check", in which information about vital signs and the degree of pain is entered; 4) Changes in physical condition that can be 


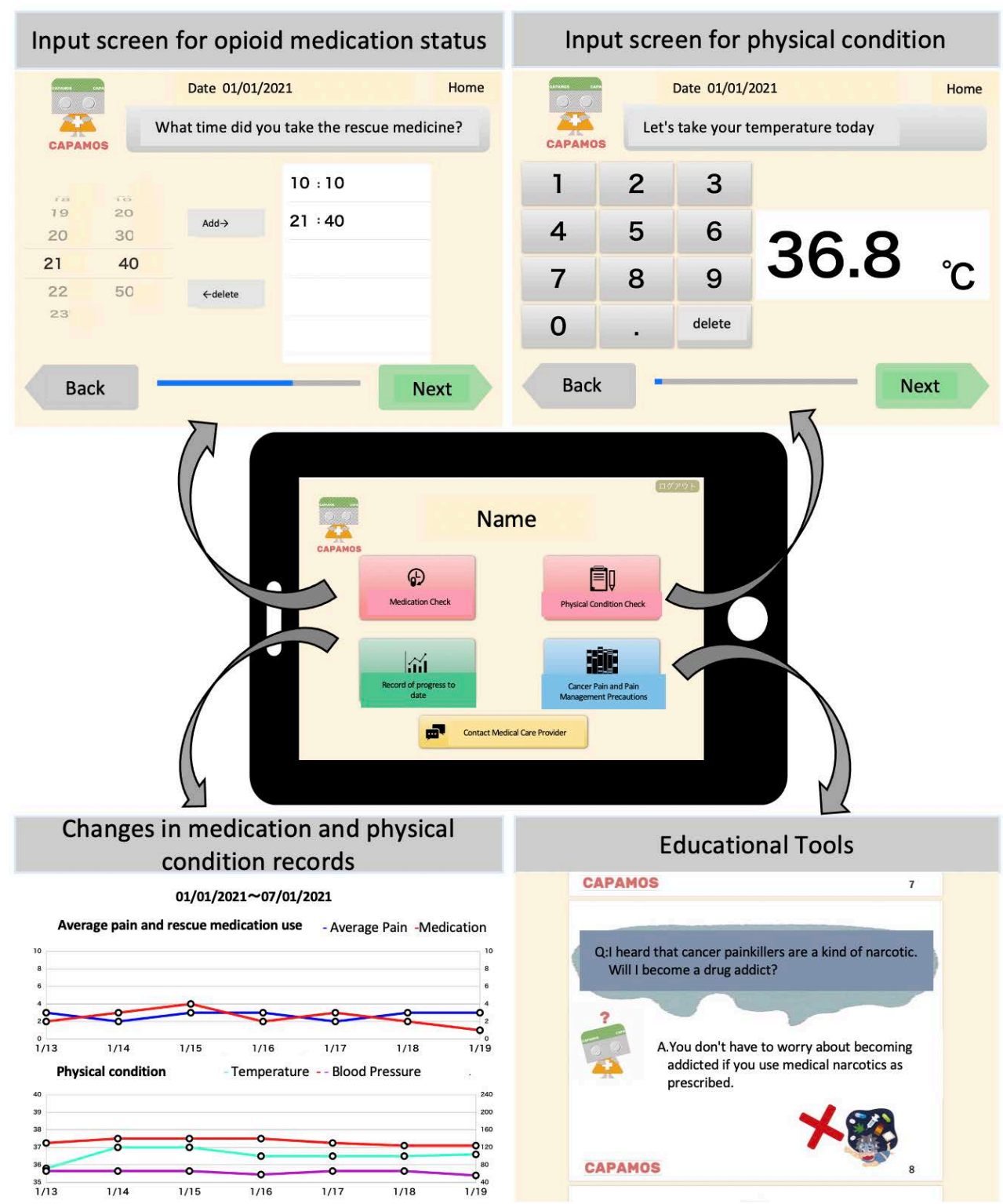

Figure 2. Components of CAPAMOS.

checked through a graph; 5) "Contact Medical Care Provider", which allows users to make video calls or send e-mails to a telenurse.

When the patient's level of pain or frequency of taking abortive medication is high, the patient's system will follow up by sending an alert notification to the healthcare provider's system. The flowchart for triggering alerts (Figure 3) was created by an oncology pharmacotherapy specialist, a palliative medicine specialist, and an oncology nurse specialist based on the National Comprehensive Cancer Network (NCCN) Guidelines (Ver 3.2019) for Adult Cancer Pain [22] and the Guidelines for Pharmacotherapy of Cancer Pain [23].

The values that trigger alert notifications are the patient's average pain intensity of NRS $\geq 7$ and the frequency of rescue medication $\geq 5$ times/day [24] [25]. The individual settings of the values that trigger alert notifications can be changed 


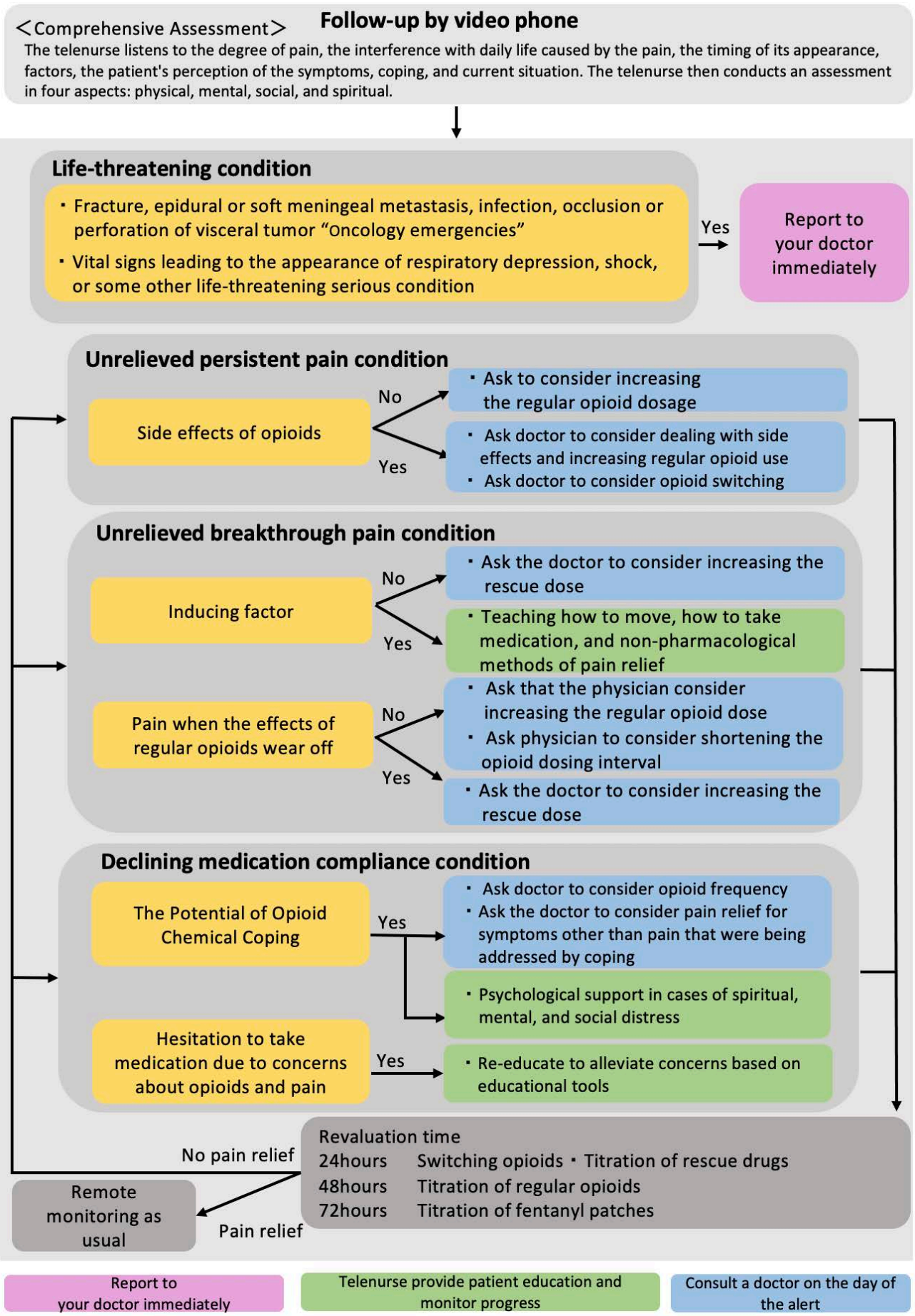

Figure 3. Flowchart for responding to alerts.

in consultation with the attending physician depending on the patient's tightening and switching status. After alert activation is confirmed, the telenurse will conduct a holistic assessment of the patient's physical, mental, social, and spiritual health via video calls. If the nurse discovers that the patient has an oncological emergency or is in a serious life-threatening situation, they will immediately report it to the attending physician. If the patient's pain is not relieved, the tele- 
nurse will assess the factors that lead to the failure of pain relief and report them to the physician. In addition, telenurse can suggest non-pharmacological measures, such as relaxation. If a patient's compliance with opioid medication is poor, re-education can be provided, or psychological support can be given if chemical coping is suspected. Re-evaluation of care measures will be performed within 24 - $72 \mathrm{~h}$ from the time the alert will be triggered. Pain will be considered to be relieved when the patient's personalized pain goal will be achieved or when the NRS will be reduced by two or more points. Thereafter, the patient's condition will be monitored.

\subsection{DATA Analysis}

The primary endpoint of cancer pain and the secondary endpoints of OSSA, BQ, EQ-5D-5L, and economic evaluation will be assessed using ANOVA or the Kruskal-Wallis test. The paired t-test or Wilcoxon test will be used to analyze the pre- and post-test values of each group. Analyses will be performed using SPSS27.0 J for Windows (IBM, NY, USA).

\subsection{Ethical Considerations}

This study was approved by the Ethics Committee of Tohoku University Graduate School of Medicine (2020-1-194). This clinical trial was registered with University hospital Medical Information Network (UMIN Clinical Trials Registry ID: 000038334). The study will be conducted in accordance with the CONSORT 2010 statement [26]. An internet protocol virtual private network (IP-VPN) with a firewall will be used to protect patients' personal information during communication. The server uses a cloud-based interface that can only be used in a closed network. Conversations with the subject will be conducted in a private telenurse monitoring center on campus to protect privacy.

\section{Discussion}

Telenursing with CAPAMOS will lead to the early detection of deteriorating pain, and patients will be able to stay at home avoiding unnecessary medical care providers. The system is equipped with an alert function to notify medical personnel about patients' abnormalities and ensure early detection. Furthermore, an ingenious aspect of this study is the creation of a telenursing algorithm that will allow telenurse to take action when an alert is triggered. In telenursing, the TNPM component of "Assessment and Communication" is important, as it allows the telenurse to gather the necessary information from the patient, determine the urgency, and act according to standardized guidelines [14] [15]. The algorithm developed in this study is based on the current guidelines [22] [23], ensuring standardized guideline-based practice. In addition, the "Evaluation of Outcomes", a component of TNPM, aims to evaluate the results of the care implemented and to repeat the care if the results were poor.

Other elements of TNPM are the "Knowledge and Experience", which refers 
to quality care and social resources, "Client Resources" that contributes toward providing resources that will yield the best results for the subject. In addition, the "Mutual Decision-making" ensures that the telenurse and the subject mutually discuss and make decisions together, and "Allocation of resource" helps in establishing a trusting relationship between the primary care provider and the subject [14] [15]. In this study, a certified nurse cancer specialist [27], who can fulfill these elements, will conduct telenursing to ensure quality. Accordingly, quality nursing care can be provided even at home, pain can be alleviated, and QOL can be maintained.

In Japan, telemonitoring was covered by medical insurance in 2018 for patients who required home oxygen therapy or continuous positive airway pressure breathing therapy [28]. However, telemonitoring of the cancer-related symptoms and pain have not been covered by medical insurance yet. This can be because of the lack of cost-benefit validation studies on telenursing and the difficulty faced by registered nurses in managing the symptoms of patients, which become more complex as the disease progresses. If the findings of this study confirm that telenursing is effective in relieving pain, it may be covered by medical insurance. CAPAMOS is a system that will be medically supervised by an oncology nurse specialist, an oncology pharmacotherapy specialist, and a palliative medicine specialist. Additionally, a certified nurse specialist in cancer nursing will be engaged as a telenurse. For these two reasons, CAPAMOS is considered capable of managing increasingly complex symptoms.

\section{Conclusion}

The RCT is expected to validate the efficacy of telenursing using CAPAMOS in patients with advanced cancer that experience pain.

\section{Source of Funding}

This study was supported by JSPS KAKENHI (grant number JP 18H06386).

\section{Conflicts of Interest}

The authors declare no conflicts of interest regarding the publication of this paper.

\section{References}

[1] Yamagishi, A., Morita, T., Miyashita, M., Mitsunori, M., Ayumi, I., Miki, A., et al. (2012) Pain Intensity, Quality of Life, Quality of Palliative Care, and Satisfaction in Outpatients with Metastatic or Recurrent Cancer: A Japanese, Nationwide, RegionBased, Multicenter Survey. Journal of Pain and Symptom Management, 43, 503-514. https://doi.org/10.1016/j.jpainsymman.2011.04.025

[2] Ministry of Health, Labour and Welfare (2012) About the Social Security and Tax Reform Charter 2012. https://www.cas.go.jp/jp/seisaku/syakaihosyou/kakugikettei/240217kettei.pdf

[3] Yoshida, S., Fumiko, S. and Naoko, S. (2019) Relationship between Cancer Pain Self-Management and Pain in Outpatients with Advanced Cancer Taking Opioid 
Analgesics. Palliative Care Research, 14, 113-126.

[4] Yoshida, S., Sato, F., Takahashi, C., Inoue, M. and Tomizawa, A. (2021) Self-Management of Opioid Analgesics for Cancer Pain Relief by Patients with Advanced Cancer at Home. Journal of Japanese Society of Cancer Nursing, 35, 254-260.

[5] Ministry of Internal Affairs and Communications (2013) ICT Super Aging Society Concept Conference Report-Realization of "Smart Platinum Society". https://www.soumu.go.jp/main_content/000226641.pdf

[6] Ministry of Health, Labour and Welfare (2014) Health/Medical Strategy. https://www.mhlw.go.jp/file/05-Shingikai-10801000-Iseikyoku-Soumuka/16_9.pdf

[7] Ministry of Health, Labour and Welfare (2016) Thinking About a Social Model that Overcomes the Aging Population-Annual Report on Health, Labor and Welfare 2016. http://www.mhlw.go.jp/wp/hakusyo/kousei/16/dl/all.pdf

[8] Galiano-Castillo, N., Cantarero-Villanueva, I., Fernández-Lao, C., Ariza-García, A., Díaz-Rodríguez, L., Del-Moral-Ávila, R., et al. (2016) Telehealth System: A Randomized Controlled Trial Evaluating the Impact of an Internet-Based Exercise Intervention on Quality of Life Pain, Muscle Strength, and Fatigue in Breast Cancer Survivors. Cancer, 122, 3166-3174. https://doi.org/10.1002/cncr.30172

[9] Donovan, H.S., Ward, S.E., Sereika, S.M., Knapp, J.E., Sherwood, P.R., Bender, C.M., et al. (2014) Web-Based Symptom Management for Women with Recurrent Ovarian Cancer: A Pilot Randomized Controlled Trial of the WRITE Symptoms Intervention. Journal of Pain and Symptom Management, 47, 218-230.

[10] Kroenke, K., Theobald, D., Wu, J., Norton, K., Morrison, G., Carpenter, J., et al. (2010) Effect of Telecare Management on Pain and Depression in Patients with Cancer: A Randomized Trial. JAMA, 304, 163-171.

https://doi.org/10.1001/jama.2010.944

[11] Basch, E., Deal, A.M., Kris, M.G., Scher, H.I., Hudis, C.A., Rogak, P.S., et al. (2016) Symptom Monitoring with Patient-Reported Outcomes during Routine Cancer Treatment. Journal of Clinical Oncology, 34, 557-565. https://doi.org/10.1200/JCO.2015.63.0830

[12] Basch, E., Deal, A.M., Dueck, A.C., Scher, H.I., Kris, M.G., Hudis, C., et al. (2017) Overall Survival Results of a Trial Assessing Patient-Reported Outcomes for Symptom Monitoring during Routine Cancer Treatment. JAMA, 318, 197-198. https://doi.org/10.1001/jama.2017.7156

[13] Yoshida, S., Sato, F., Tagami, K., Shimoyama, M. and Takahashi, S. (2021) Evaluating the Pilot Usability for Telenursing-Based Cancer Pain Monitoring System. Palliative Care Research, 16, 99-108.

[14] Larson-Dahn, M.L. (2000) Tel-eNurse Practice: A Practice Model for Role Expansion. JONA: The Journal of Nursing Administration, 30, 519-523. https://doi.org/10.1097/00005110-200011000-00006

[15] Larson-Dahn, M.L. (2001) Tel-eNurse Practice. Quality of Care and Patient Outcomes. JONA: The Journal of Nursing Administration, 31, 145-152. https://doi.org/10.1097/00005110-200103000-00011

[16] Dodd, M., Janson, S., Facione N., Faucett, J., Froelicher, E.S., Humphreys, J., et al. (2001) Advancing the Science of Symptom Management. Journal of Advanced Nursing, 33, 668-676. https://doi.org/10.1046/j.1365-2648.2001.01697.x

[17] Oldenmenger, W.H., Baan, M.A.G. and van der Rijt, C.C.D. (2018) Development and Feasibility of a Web Application to Monitor Patients' Cancer-Related Pain. Supportive Care in Cancer, 26, 635-642. https://doi.org/10.1007/s00520-017-3877-3

[18] Uki, J., Mendoza, T., Cleeland, C.S., Nakamura, Y. and Takeda, F. (1998) A Brief 
Cancer Pain Assessment Tool in Japanese: The Utility of the Japanese Brief Pain Inventory BPI-J. Journal of Pain and Symptom Management, 16, 364-373.

[19] Kondo, Y. and Shibuya, S. (2002) Concerns about Morphine and Medication Behavior in Cancer Outpatients with Pain. Journal of Japanese Society of Cancer Nursing, 16, 5-16.

[20] Ikeda, S., Shiroiwa, T., Igarashi, A., Noto, S., Fukuda, T., Saito, S., et al. (2015) Developing a Japanese Version of the EQ-5D-5L Value Set. Journal of the National Institute of Public Health, 64, 47-55.

[21] Ruland, C.M., Andersen, T., Jeneson, A., Moore, S., Grimsbø, G.H., Børøsund, E., et al. (2013) Effects of an Internet Support System to Assist Cancer Patients in Reducing Symptom Distress: A Randomized Controlled Trial. Cancer Nursing, 36, 6-17. https://doi.org/10.1097/NCC.0b013e31824d90d4

[22] Swarm, R.A., Paice, J.A., Anghelescu, D.L., Are, M., Bruce, J.Y., Buga, S., et al. (2019) Adult Cancer Pain, Version 3.2019, NCCN Clinical Practice Guidelines in Oncology. Journal of the National Comprehensive Cancer Network, 17, 977-1007.

[23] Japanese Society for Palliative Medicine, Palliative Medicine Guidelines Preparation Committee (2014) Guidelines Related to Pharmacotherapy for Cancer Pain. Kanehara Publishing, Tokyo, 212-219.

[24] Tagami, K., Okizaki, A., Miura, T., Watanabe, Y.S., Matsumoto, Y., Morita, T., et al. (2018) Breakthrough Cancer Pain Influences General Activities and Pain Management: A Comparison of Patients with and without Breakthrough Cancer Pain. Journal of Palliative Medicine, 21, 1636-1640.

https://doi.org/10.1089/jpm.2017.0675

[25] Davies, A., Buchanan, A., Zeppetella, G., Josep, P.S., Rudolf, L., Wolfgang, W., et al. (2013) Breakthrough Cancer Pain: An Observational Study of 1000 European Oncology Patients. Journal of Pain and Symptom Management, 46, 619-628. https://doi.org/10.1016/j.jpainsymman.2012.12.009

[26] Moher, D., Hopewell, S., Schulz, K.F., Montori, V., Gøtzsche, P.C., Devereaux, P.J., et al. (2012) CONSORT 2010 Explanation and Elaboration: Updated Guidelines for Reporting Parallel Group Randomized Trials. International Journal of Surgery Open, 10, 28-55. https://doi.org/10.1016/j.ijsu.2011.10.001

[27] Japanese Nursing Association (2021) About Certified Nurse Specialist. https://nintei.nurse.or.jp/nursing/qualification/probation_guide_cns

[28] Ministry of Health, Labour and Welfare (2016) About Utilization of ICT in Medical Care. https://www.niph.go.jp/h-crisis/wp-content/uploads/2019/06/000517305.pdf

\section{List of Abbreviations}

BPI-J, Japanese Brief Pain Inventory; BQ, Barriers Questionnaire; CAPAMOS, Cancer Pain Monitoring System; ELNEC-J, end-of-life nursing education consortium-Japan; IP-VPN, internet protocol virtual private network; MSM, model of symptom management; NCCN, National Comprehensive Cancer Network; NRS, numerical rating scale; OSSA, Opioid Self-Management Scale for Advanced Cancer Patients; RCT, randomized controlled trial; TNPM, Tel-eNurse Practice Model; QOL, quality of life. 